\title{
A Wireless Medical Monitoring Over a Heterogeneous Sensor Network
}

\author{
Mehmet R. Yuce, Peng Choong Ng, Chin K. Lee, Jamil Y. Khan, and Wentai Liu
}

\begin{abstract}
This paper presents a heterogeneous sensor network system that has the capability to monitor physiological parameters from multiple patient bodies by means of different communication standards. The system uses the recently opened medical band called MICS (Medical Implant Communication Service) between the sensor nodes and a remote central control unit (CCU) that behaves as a base station. The CCU communicates with another network standard (the internet or a mobile network) for a long distance data transfer. The proposed system offers mobility to patients and flexibility to medical staff to obtain patient's physiological data on demand basis via Internet. A prototype sensor network including hardware, firmware and software designs has been implemented and tested by incorporating temperature and pulse rate sensors on nodes. The developed system has been optimized for power consumption by having the nodes sleep when there is no communication via a bidirectional communication.
\end{abstract}

\section{INTRODUCTION}

With the advancement of wireless technologies, wireless sensor networks can greatly expand our ability to monitor and track conditions of patients in healthcare area. High performance and fault tolerant wireless devices can now be employed to eliminate medical errors, to reduce workload and increase the efficiency of hospital staff, and to improve the comfort of patients. Thus, there has been increased interest among research groups in developing wireless recording and monitoring for real-time physiological parameters (e.g. ECG, EEG, EOG, EMG, Neural, Blood Flow, Blood Pressure etc.) from a patient body in medical environments [1]-[6]. Existing wireless data collection systems use standards such as ZigBee (IEEE 802.15.4) or Bluetooth (IEEE 802.15.1). Those standards have some drawbacks as they do not exactly comply with the medical standard due to their size, power consumption and strong interference from other devices in medical environment. Considering hundred sensors attached to a patient's body, such systems become quite bulky to be carried by patients.

International communications authorities have recently allocated a new band Medical Implant Communication Service (MICS) band at $402-405 \mathrm{MHz}$ with ten $300 \mathrm{KHz}$ channels to enable the wireless communication of medical devices to deliver high level of comfort, mobility and better patient care [7][8]. In addition, the 402-405 MHz frequencies have propagation characteristics conducive to the transmission of radio signals within human body [9].

Manuscript received April 16, 2007. This work was supported in part by the Australian Research Council (ARC) under Discovery Projects Grant.

M. R. Yuce, P. C. Ng, C. K. Lee, and J. Y. Khan are with the School of Electrical Engineering and Computer Science, University of Newcastle, Callaghan, NSW 2308, Australia(e-mail: mehmet.yuce@newcastle.edu.au ).

W. Liu is with the Electrical Engineering Department, University of California, Santa Cruz, CA 95064 USA
The aim of this project is to build a Wireless Body Sensor Network (WBSN) that is based on the newly available 402$405 \mathrm{MHz}$ MICS band. The project targets both implanted and on-body (i.e. external) nodes. Instead of applying other standards such as Bluetooth, ZigBee or WLANs for data collections from sensors, this band was particularly chosen in our design to eliminate the strong interference from other devices as reliable communication and accurate monitoring are very crucial for patients' lives. The MICS do not also cause interference to other users of the electromagnetic spectrums [11]. Another advantage is that when nodes used as implantable, it provides small size, low-power, faster data transfer and longer communication range [7][8][13 Combining all these features with the acceptance of the 402$405 \mathrm{MHz}$ band internationally offers an attractive frequency choice for the targeted WBSN application.

Previously we reported a MICS based WBSN in [14] that has the capability of simplex communication and only allows one patient's condition to be monitored locally. In this work we present a multi-patient monitoring system with data transfer ability over a network or the internet to a remote computer. Hardware and software designs have been developed to reach our goals. A media access layer (MAC) has been successfully implemented to support multi-patient monitoring facility. Moreover, data accessibility has been successfully extended to a remote computer that maybe located within a local area network (i.e. LAN) or to the internet. Hardware modules have been designed to support both indoor and outdoor environments which means that a patient can be either in a medical center or in his/her house or an outside location that is close to the internet or a mobile communication network.

\section{Multi-Patient Body Sensor Network System}

The proposed WBSN overview is as shown in Fig. 1. This wireless body sensor network comprises of sensor nodes, a Central Control Unit (CCU) that transmits data to a local PC and a receiver station at a medical center. Sensor nodes are responsible of obtaining raw data from a human body. After processing, they transmit those data to the CCU via the wireless RF link using the MICS band. The CCU then repackages the data and transmits to the local PC. The data collected at the local PC is transferred to a remote PC across the network in a medical center or through internet if it is at a different location than the medical center. Currently as a part of our future work, a second wireless link aiming a Bluetooth communication between the CCU and a mobile device is underdevelopment. Such a wireless link will provide a further mobility to patients as they can go out to continue their normal routine life. 


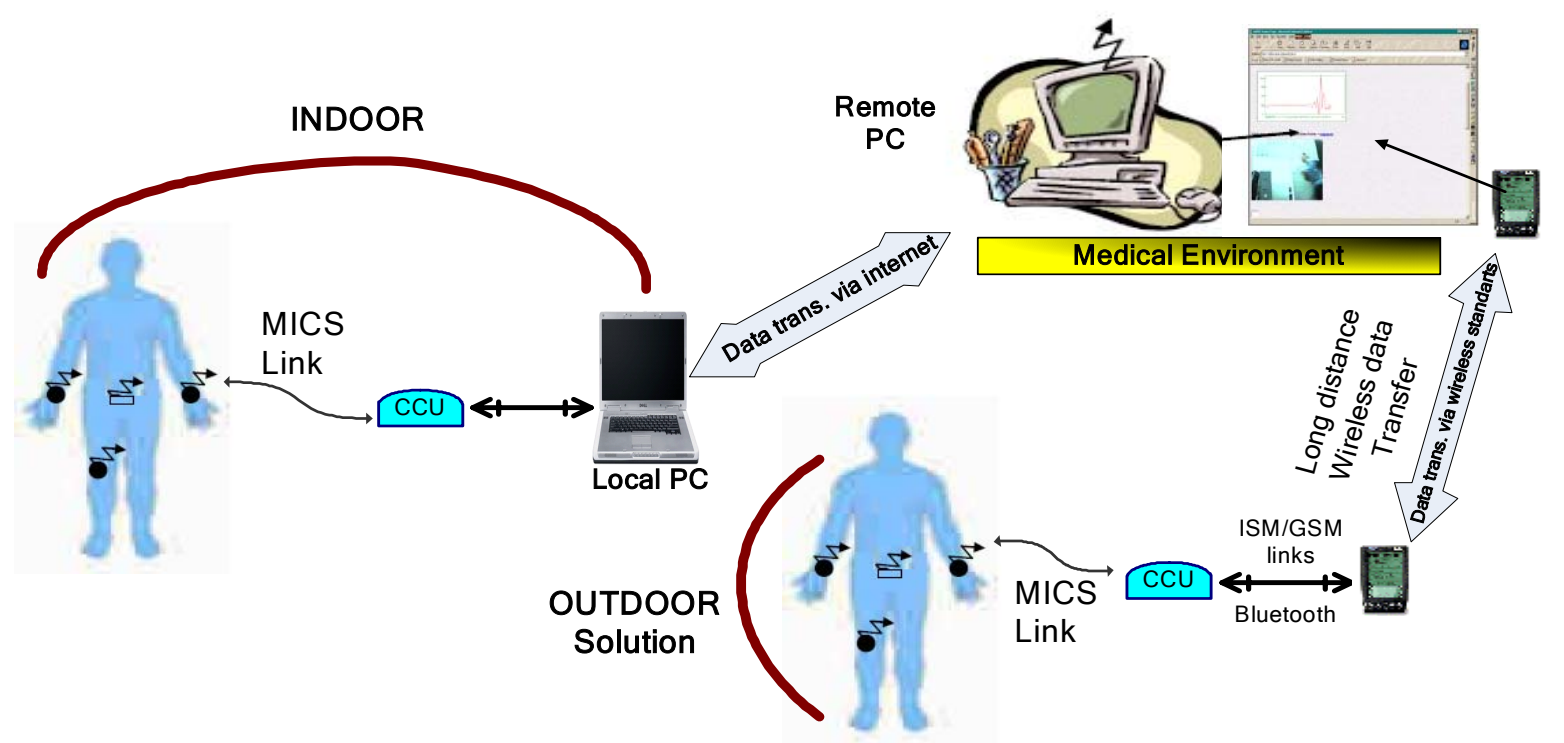

Figure-1. A heterogeneous wireless network system for medical monitoring.

The receiver station (i.e. the remote PC) is capable of displaying all the received data on a User Display Graphic (GUI) and is also capable of storing all the data in the database system of a medical center. The $\mathrm{CCU}$ is targeted to be worn around a patient's waist. For the patients with limited mobility, the CCU will be replaced at an accessible location at a distance of up to 10 meter in a house. In the later case, the $\mathrm{CCU}$ is connected to a local PC that displays the real-time information received from the sensor nodes and records the information to a database locally, the same way that is done at the remote $\mathrm{PC}$ of the medical center. The stored information can be sent via internet to the database of the medical center on a periodic basis. When more than one patient is accommodated in a room as in the case of a hospital, the necessary software packages that are explained later in section IV ((See Fig 5.) are installed in the CCU and the local $\mathrm{PC}$ in order to obtain physiological signals from sensors of each patient. Data gathering is performed at some certain time intervals assigned to patients. Pulse rate and temperature are the two vitals signs selected to be monitored in the prototype system to demonstrate a multi user network.

\section{SENSOR NODES AND CCU HARDWARE DESIGNS}

Sensor nodes are designed to collect raw signals from a human body. The signal from a human body is usually weak and coupled with noise. First, the signal should go through amplification and filtering process to increase the signal strength, and to remove unwanted signals and noise. After which, it will go through an Analog to Digital conversion (ADC) stage to be converted into digital for digital processing. The digitized signal is then processed and stored in the microprocessor. The microprocessor will then pack those data and transmit over the air via a transmitter (see Fig. 2). The overview of a Pulse Rate Sensor Node is shown in Fig. 2 [14]. As can been seen, the pulse rate sensor node comprises of. Microcontroller PIC16F877 and the transceiver AMIS-5210 are selected in the project because of the following reasons: low-power consumption, size, and the suitability operating at the MICS band and for the physiological data processing [14]. Fig. 3 shows the hardware implementation of sensor nodes. Both temperature and pulse rate sensor nodes were built on a common PCB circuit. Thus the electronics can be used interchangeable. The antennas for this project are designed as a loop printed around the prototyping boards (See Fig. 3). Hardware implementation of the Pulse Rate Sensor Node is shown in Fig. 3.

The CCU also requires a micro-controller and a wireless transceiver chip to coordinate all activities similar to the sensor nodes. The CCU hardware is made of the same transceiver chip from AMI semiconductor (AMI52100 IC) and the microcontroller PIC16F87 [14]. The targeted wireless distance between sensors and the CCU (the MICS link) is 110 meters. The CCU can thus be located at the waist of the patient or at an easily accessible place.

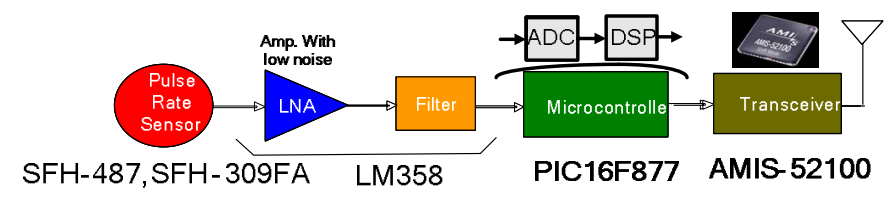

Figure-2. Block diagram of a pulse rate sensor node.

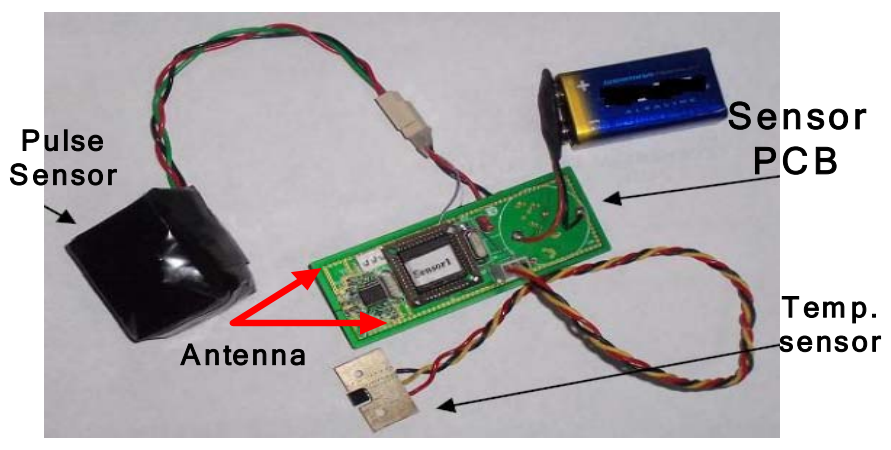

Figure-3. Hardware design of sensor nodes. 


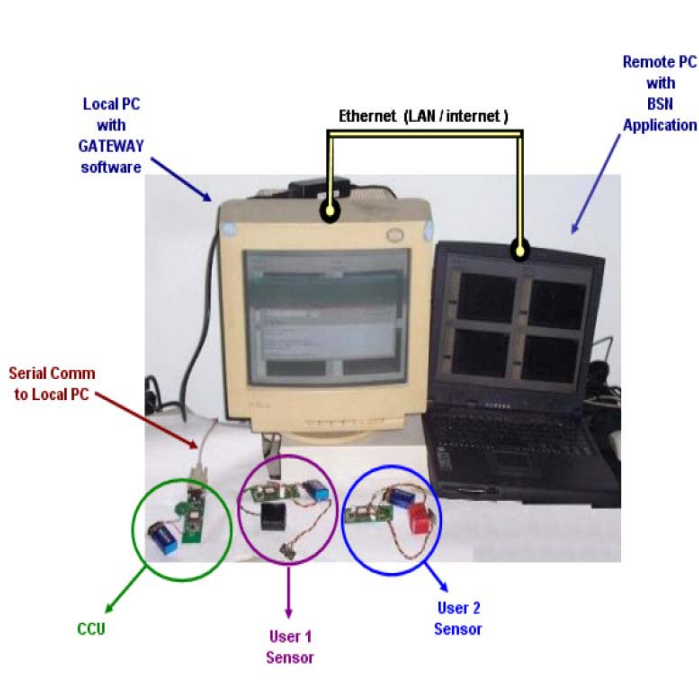

Figure-4 Set up of the multi-patient body sensor network system.

\section{Data Base, Software Programs and Monitoring}

Since all sensor nodes of a body are communicating with the same CCU, the data is prefixed with an identifier that is used to identify source of data. To reduce collisions further between data sent, a firmware (media access layer (MAC) protocol) is written to control data transmissions. The communication between the sensors and the CCU is bidirectional as to support a multi user (i.e. multi-patient) communications. To illustrate a multi user implementation, we need at least 2 users with two sensors (each user uses two sensors) and one CCU for the test and demonstrations of the extensive software packages developed. The experimental setup is given in Fig 4.

A media access layer (MAC) protocol for firmware at both sensor node and the $\mathrm{CCU}$ is developed to prove the bidirectional communication. The system was designed using the CSMA/CA (Carrier Sense Multiple Access with Collision Avoidance) MAC protocol to accommodate multi-patient medical data transfer. In this design we used the RTS/CTS (Ready To Send/Clear To Send) feature of the MAC protocol for reliable transfer of the medical records. When a sensor node wants to send data to the CCU first it will check the transmission channel. If the node finds the channel is free then it will transmit a RTS packet otherwise the node will backoff for a future transmission attempt. On successful reception of the RTS packet the CCU will send a CTS packet confirming the acceptance of the transmission. By reading the CTS packet information other nodes will remain in quite state allowing the transmitting node to capture the channel and transmit its own information. The transmitting node will release the channel as soon as the transmission of information packet is complete. This simple MAC protocol allows multiple sensor nodes to reliably transmit their data to the CCU without any significant delay.
Figure-5. Overview of programs used for communications, a) when the device used individually, or b) for multi-patient in medical centers.

A software (defined as GATEWAY) has been developed at the local PC to communicate with the $\mathrm{CCU}$ to get readings from sensors and then forward them through the network/internet to an application on a remote PC (at medical center). While performing this task, the GATEWAY also verifies the data integrity and schedules retransmission if required. Another software program is developed at the remote PC (called BSN) who gets readings from GATEWAY via network/internet. These readings are stored in the remote PC for analysis. The program is also able to display readings for multiple users. Interaction between software programs is depicted in Fig. 5. Fig. 6 shows a graphical user interface (GUI) at the local PC that is designed to configure the source and destination socket and port numbers for data transfer on

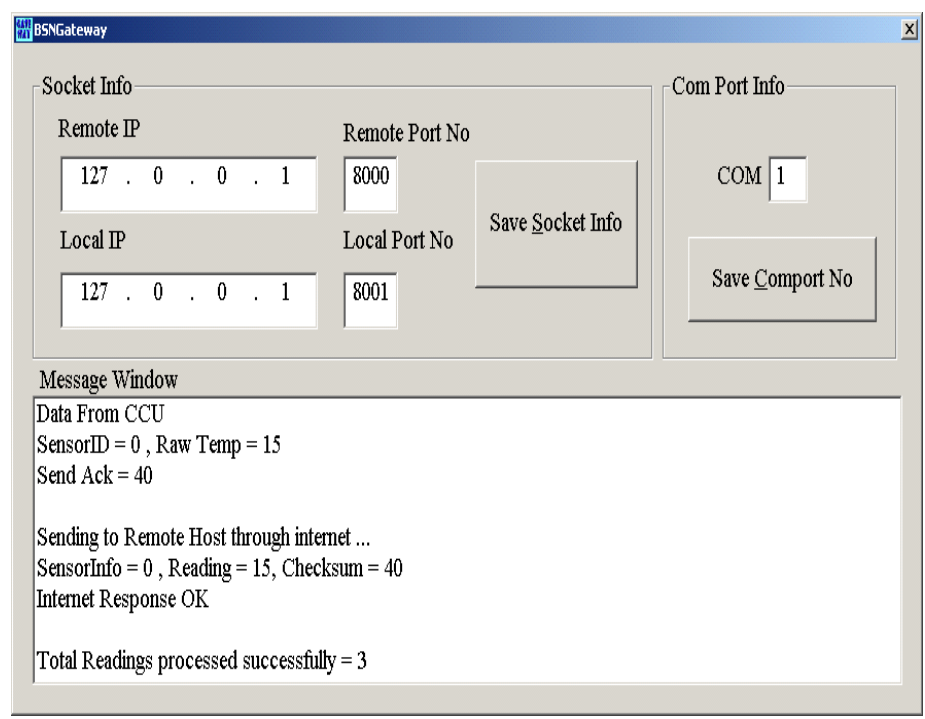

Figure-6 GATEWAY menu. 


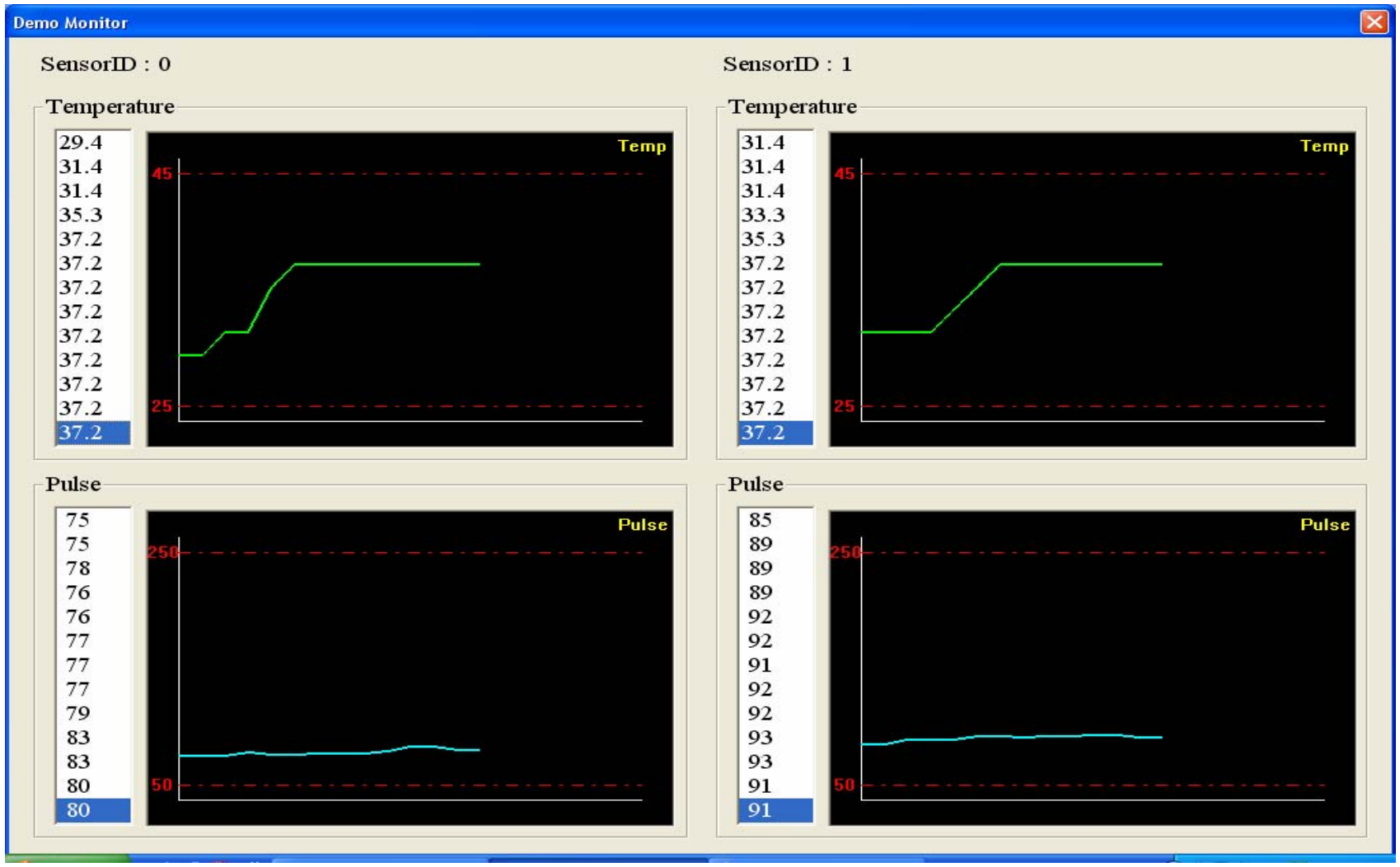

Fi gure-7. Live monitoring of multi-patients.

the Ethernet. This GUI ensures the portability if BSN application or GATEWAY need to be relocated, it can be configured to work without additional changes in the codes. Both the data received from the CCU and the data sent to the BSN can also been shown here in a text format.

The BSN application is designed so as to collect and store readings automatically so that no person is required to be stationed at the application. It takes care of the administration of patients' particulars such as assigning new sensor ID to patients, segregating sensor readings from different patients and storing them into the data base. The GUI also allows the medical personnel to enter the patient's information. It can also display the live monitoring graphs on the screen. An example of live monitoring from our set up is shown in Fig. 7. It displays temperature and pulse rate information of two patients at the same time. The graph allows up to 20 readings to be displayed. Every sensor device has a unique Sensor ID and must be registered under a patient name before they are used. In the event that an unregistered sensor node is used, all its readings received will be discarded by the BSN application.

\section{Performance Evaluation}

A test was conducted to evaluate the bit-error rate (BER) performance of the wireless transmission with the distance. The BER indicates the reliability of a channel. For the test, 625000 bytes $\left(5 \times 10^{6}\right.$ bits $)$ of data were sent continuously from a sensor node to the CCU. If any bit of the received data is in error, the whole 8 bits in the byte were considered to be in error. The BER versus the distance is depicted in Fig. 8. As the distance increases the error rate increases since the transmitter signal is getting weak.

Another measurement shown in Fig. 9 has been done to evaluate the throughput performance of the network. Error checking mechanisms (i.e. Checksum) are included in the software designs mentioned in the previous section in order to provide a retransmission if a corrupted reading (sensor information) is received. With the 1000 bytes of data sent in the measurement, the throughput of the MAC layer is measured as the number of readings in a second. Fig. 9 shows the graph of throughput versus distance with four different configurations: one patient-CCU communication with and without error checking and two patients-CCU communication with and without error checking algorithms. Throughput for one user under the MAC is slightly higher than that with 2 Users. The throughput with Checksum is also slightly lower than that without Checksum as additional checksum transmitted incur more transmission time and thus a lower result.

The transmitter power for the MICS band is limited by the regulation which can be chosen maximum $25 \mu \mathrm{W}$ (i.e. $E I R P=-16 \mathrm{dBm})[7][8]$. This is the radiated power at the output of the transmitter antenna used in our setup. The transmission range is basically limited by the receiver sensitivity of the AMIS transceiver [10]. The sensor nodes are able to transmit data to the CCU up to $12 \mathrm{~m}$ range [14]. . 


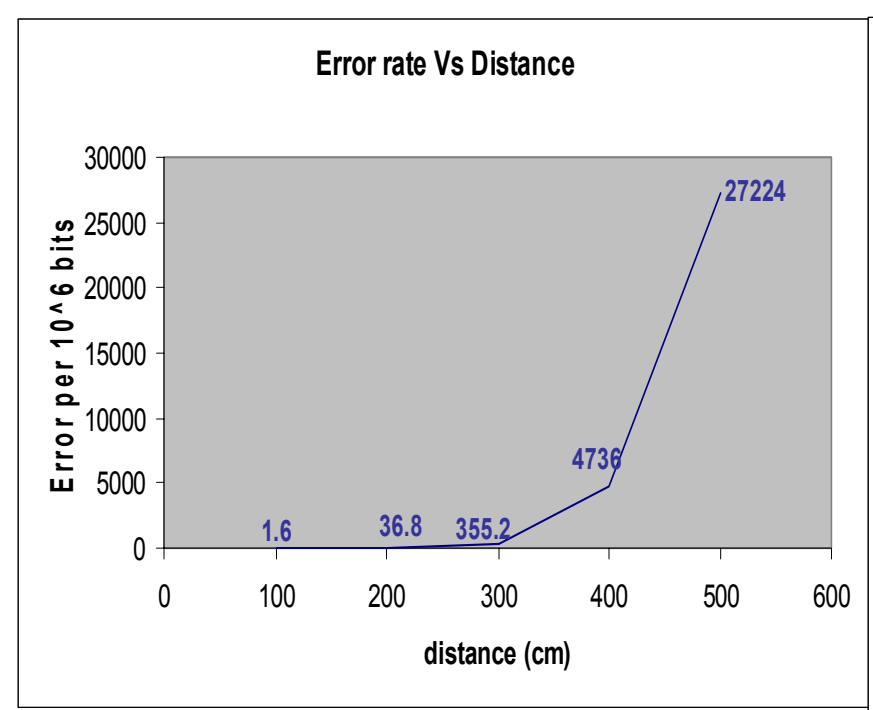

Fig ure-8. BER with distance

The sensors are calibrated for data accuracy before usage. The temperature sensor is calibrated with a Mini-Logger (GL500) while the pulse rate sensor is calibrated with a commercial pulse rate sensor approved by health authorities. Under the Live-Monitoring Mode (i.e. continuously obtaining readings from sensors), the total current consumption of the sensor hardware with both sensors attached is 126 $\mathrm{mA}$. Using a battery of 560mAh (coin battery CR2450), the battery last 4.44 hours. The power consumption is further reduced by keeping sensor nodes in sleep mode for longer durations (i.e. increasing off-time)[14].

\section{Conclusion}

A wireless sensor network system for monitoring physiological parameters from multiple patient bodies has been developed. A prototype system that is able to acquire readings from multiple patients has been presented. It has been shown that after obtaining data from sensors, the data can be transferred to a remote PC through a local area network or the internet for further analysis. Such a wireless body sensor network system is very suitable to be used in hospitals environments. Human errors will be reduced and health professionals will spend their time more on other important issues. In addition, such systems result in an increase in patients' comfort level as they no longer need to be wakening up for periodic checks in the hospital environments. Another advantage is that patients can stay in their home while their conditions can still be monitored by medical staff. More wards could thus be available to patients in medical centers.

Future work also involves in including more sensors to monitor other vital signs such as blood pressure, oxygen saturation level, ECG, etc. The system can also include the evaluation of physiological parameters by the software package at the local pc before transmitted to the medical center. There is also possibility to extend the capability to automate alerts which can get attention of the health professional as soon as they are needed.

\section{Throughput vs Distance}

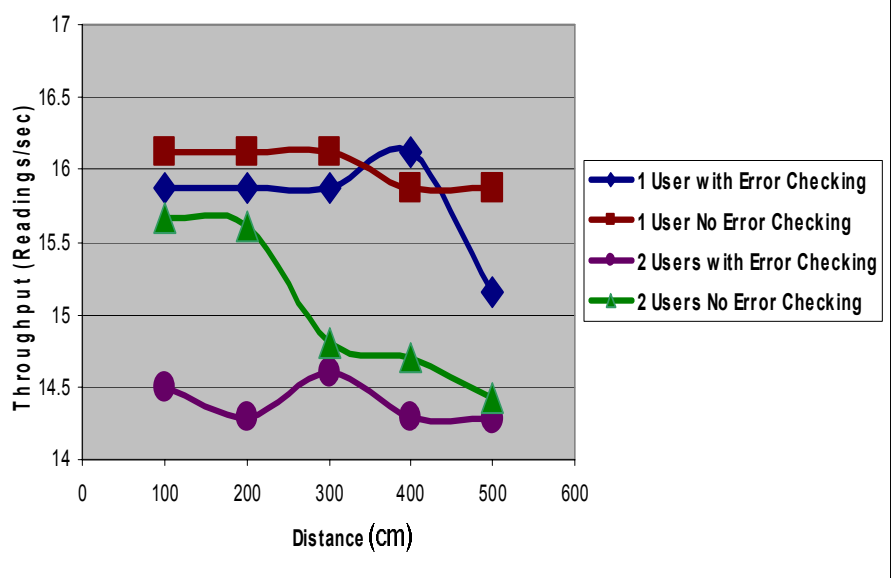

Figure-9. Throughput vs distance

\section{REFERENCES}

[1] C. C. Y. Poon, Y-T Zhang, S-D Bao, "A novel biometrics method to secure wireless body area sensor networks for telemedicine and $\mathrm{m}$ health," IEEE Communications Magazine, vol. 44, pp. 73-81, April 2006 .

[2] R. Fischer et al. SMART: Scalable Medical Alert and Response Technology. http://smart.csail.mit.edu/.

[3] T. Gao et al. "Vital Signs Monitoring and Patient Tracking Over a Wireless Network," IEEE-EMBS 27th Annual Int. Conference of the Eng. in Medicine and Biology, Sept. 2005, Page(s):102 - 105.

[4] U. Anliker, "AMON: a werable multiparameter medical monitoring and alert system, in IEEE Trans. Information Tech. In Biomedicine, vol. 8, Dec 2004.

[5] F. Magrabi, N. H. Lovell, and B. G. Celler, "Web based longitudinal ECG monitoring," Proc. 20th Annu. Int. Conf. IEEE EMBS, vol. 20, no. 3, pp. 1155-1158, 1998.

[6] S. Park, et al., "Real-time monitoring of patient on remote sites," Proc. 20th Annu. Int. Conf. IEEE EMBS, vol. 20, no. 3, pp. 1321-1325, 1998.

[7] FCC Rules and Regulations, "MICS Band Plan," Table of Frequency Allocations, Part 95, Jan. 2003.

[8] Australian Communications Authority, Radio Frequency Planning Group, "Planning for Medical Implant Communications Systems \& Related Devices", Proposals Paper, Oct. 2003.

[9] W. G. Scanlon, J. B. Burns, and N. E. Evans, "Radiowave propagation from a tissue implanted source at $418 \mathrm{MHz}$ and 916. $5 \mathrm{MHz}$," IEEE Trans. Biomedical Engineering, vol. 47, pp.527-534, April 2000.

[10] AMIS 52100 Low Power Transceiver with Clock and Data Recovery Data Sheet.

[11] http://www.wirelessis.com, April 2007.

[12] A. Tekin, M. R. Yuce, and W. Liu, "A Low power MICS band transceiver architecture for implantable devices," in Proc. IEEE Wireless and Microwave Technology Conference, pp. 55-58, April 2005.

[13] A. Tekin, M. R. Yuce and W. Liu, "Integrated VCO Design for MICS Transceivers," in Proc. IEEE Custom Integrated Circuits Conference, pp. $765-768$, Sept. 2006.

[14] M. R. Yuce et al. "A MICS wireless body sensor network" in IEEE Wireless Communications and Networking Conference (WCNC), pp. 2473-2478, March 2007. 